\title{
IS THERE A CONNECTION BETWEEN THERMAL PULSES AND PNE HALOS: AN APPROACH TO AN ANSWER
}

\author{
ADAM FRANK and BRUCE BALICK \\ Astronomy Department, FM-20, University of Washington, Seattle WA 98195, USA \\ and \\ WILL VAN DER VEEN \\ Astronomy Department, Columbia University, N.Y., N.Y. 10027, USA
}

\begin{abstract}
We wish to explore the hypothesis that the shells and halos of planetary nebulae are formed during the thermal pulsing of progenitor AGB stars. Using published data and model results we compare the AGB interpulse time $\Delta \mathrm{t}_{\mathrm{T}}$ and the time between shell ejections $\Delta \mathrm{t}_{\mathrm{D}}$ for a sample of PNe with halos. $\Delta \mathrm{t}_{\mathrm{T}}$ is derived from the Paczynski relation using the PNe central star luminosity. $\Delta t_{D}$ is calculated using the radii and velocity of PNe shells and halos.

Conclusions. 1. 5 out of 6 of the PNe in fig 1 have $\Delta t_{D}$ and $\Delta t_{T}$ that are within an order of magnitude of each other. 2. The analysis is extremely sensitive to the assumed distances as the two points for NGC 7662 demonstrate. 3. One must be careful not to consider PNe central stars that are already on cooling tracks. The $\Delta \mathrm{t}_{\mathrm{T}} \mathrm{L}_{*}$ relation does not hold for these cases. This seems to apply to NGC 6720. 4. All the points lie close to or above the $\Delta t_{D}=$ $\Delta \mathrm{t}_{\mathrm{T}}$ line but never below it.

After removing NGC 6720 out of the plot and considering the different luminosities and distances quoted for NGC 7662, there is only one point more than $1 \sigma$ from the line $\Delta t_{D}=$ $\Delta t_{T}$
\end{abstract}

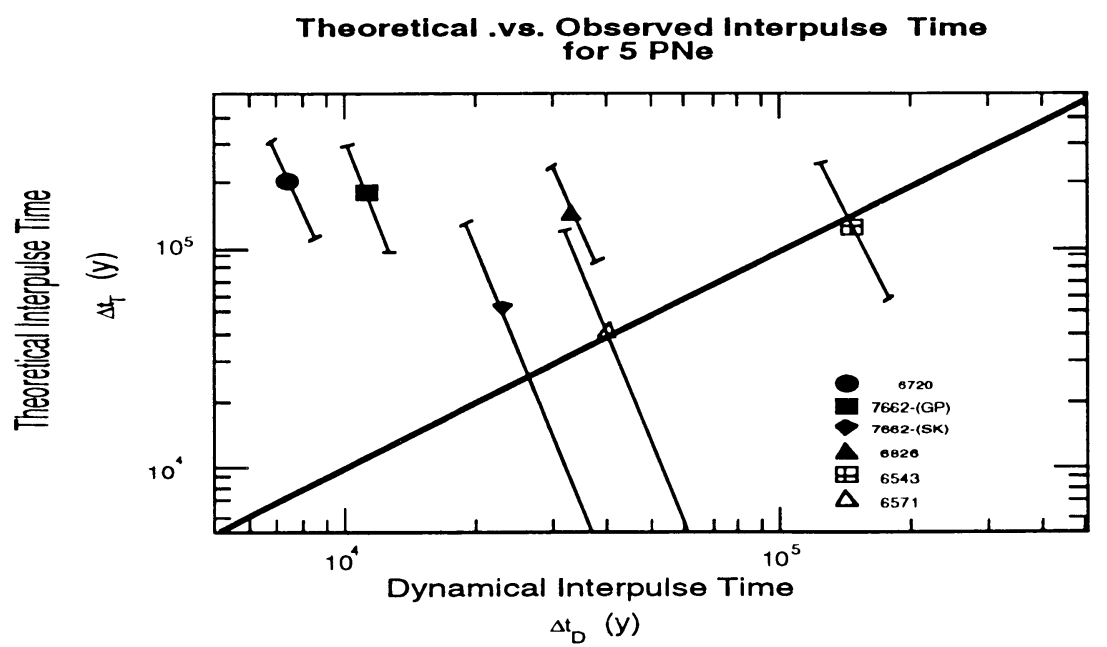

Fig 1. Data points are labeled with their NGC numbers. We used data from two authors for NCG 7662. 